\section{Plutonium particles: some like them hot}

The Medical Research Council's 1975 report The Toxicity of Plutonium (Nature, $253,385)$ concludes that "there is no evidence that irradiation by 'hot particles' in the lung is markedly more hazardous than the same activity uniformly distributed or that the currently recommended standards for inhalation of plutonium are seriously in error." Report R29 (1974) of the National Radiological Protection Board (NRPB) states that "there is no biological evidence available at present which suggests that "hot spots' carry a higher risk of cancer induction." But in the March 20, 1975 New Scientist, Dr A. R. Tamplin warns: "The plutonium exposure standards ... must and will eventually be made more restrictive by a factor approaching 1,000." Behind this disagreement is a major controversy over the risk of lung cancer from inhaled insoluble particles of intensely radioactive alpha emitters such as plutonium. In that controversy-unresolved by any direct data-lies a source of uncertainty about the future of nuclear power. A report by Amory B. Lovins and Walter C. Patterson of Friends of the Earth.
SINCE the late 1960s, many expertsGeesaman, Langham, Long, Morgan among others - have expressed concern about the formidable but highly uncertain toxicity of plutonium $(\mathrm{Pu})$ aerosols. On February 14, 1974, Drs Tamplin and Cochran of the Natural Resources Defense Council (NRDC) took action. They petitioned the United States authorities to reduce the maximum permissible lung burden (MPLB) and maximum permissible concentration in air $\left(\mathrm{MPC}_{\mathrm{a}}\right)$ of insoluble $\mathrm{Pu}$ by 115,000 times. Also in 1974, the US Atomic Energy Commission published two defences of present standards, LA-5482-PR and WASH-1320, and in May and November NRDC published interstitial rebuttals to which the AEC declined to respond in writing or otherwise. The NRDC petition is under review by many US official bodies, and the AEC's successor agency should announce its next move in April.

The cancer risk from microscopic inhaled particles of insoluble $\mathrm{Pu}$ and similar actinides arises because such particles can deposit in deep lung tissue, can stay there for a year or more, and, being hard alpha emitters, can irradiate only that tissue which is within $\sim 40-45$ $\mu \mathrm{m}$. This last property makes it hard to determine the MPLB and $\mathrm{MPC}_{a}$, for these are derived from an international standard which says merely that lungs should not be exposed to more than 15 rem of radiation per year. Fifteen rem is equivalent to $1.5 \mathrm{rad}$ of alpha radiation (since alpha radiation is considered 10 times as carcinogenic as gamma radiation), and a rad, the basic unit of "radiation absorbed dose", is that amount of radiation which deposits $100 \mathrm{erg}$ of energy per gram of tissue irradiated. But which tissue is that? Present practice is to average the alpha dose over the entire 1000 -g pair of lungs whether it all receives alpha radiation or not.

This practice is illustrated in Table 1, which shows approximate data for some hypothetical distributions of one MPLB of the dominant isotope ${ }^{239} \mathrm{Pu}(16 \mathrm{nCi}=$ 0.016 microcurie, the amount whose
Table 1 Some illustrative and hypothetical uniform distributions of $16 \mathrm{nCi}$ (=1 MPLB) of ${ }^{239} \mathrm{PuO}_{2}$ in $1 \mathrm{~kg}$ of lung tissue

\begin{tabular}{|c|c|c|c|c|c|}
\hline Number of particles & 1 & 5 & $5 \times 10^{4}$ & $1.5 \times 10^{7}$ & $5 \times 10^{7}$ \\
\hline Particle diameter $(\mu \mathrm{m})$ & 37 & 10 & 1 & 0.15 & 0.1 \\
\hline Particle mass (ng) & 300 & 6 & 0.006 & $2 \times 10^{-5}$ & $6 \times 10^{-6}$ \\
\hline Particle activity (pCi) & 16,000 & 300 & 0.3 & 0.001 & $3 \times 10^{-4}$ \\
\hline Notional lung mass & & & & & \\
\hline $\begin{array}{l}\text { irradiated } \\
\text { Fraction of } 1 \mathrm{~kg}\end{array}$ & $\begin{array}{c}65 \mu \mathrm{g} \\
7 \times 10^{-8}\end{array}$ & $\begin{array}{r}3.3 \mathrm{mg} \\
3 \times 10^{-6}\end{array}$ & $3.3 \mathrm{~g}$ & $1 \mathrm{~kg}$ & $1 \mathrm{~kg}$ \\
\hline Local tissue dose & $200 \mathrm{Mrem} / \mathrm{yr}$ & $4 \mathrm{Mrem} / \mathrm{yr}$ & $4 \mathrm{krem} / \mathrm{yr}$ & $15 \mathrm{rem} / \mathrm{yr}$ & $15 \mathrm{rem} / \mathrm{yr}$ \\
\hline $\begin{array}{l}\text { Mean dose to } 1 \mathrm{~kg} \text { lung } \\
\text { tissue }\end{array}$ & $15 \mathrm{rem} / \mathrm{yr}$ & $15 \mathrm{rem} / \mathrm{yr}$ & $15 \mathrm{rem} / \mathrm{yr}$ & $15 \mathrm{rem} / \mathrm{yr}$ & $15 \mathrm{rem} / \mathrm{yr}$ \\
\hline
\end{tabular}

The distributions shown in the last two columns give a local-tissue dose equal to the average lung dose because the radiation fields of that many particles must overlap, whereas in the first three columns, the radiation fields from so few uniformly distributed particles are insufficient to "fill" the lung. In reality, the distribution would not be uniform, and the latter position would arise with particles smaller than shown in the last two columns. Two further points: about half the energy of the alpha radiation is dissipated in the first $20 \mu \mathrm{m}$ of tissue radius, or in about $1 / 8$ of the volume irradiated by each particle; and since some attenuation of the alpha radiation in a spongy lung occurs in air rather than in tissue, the mass of tissue irradiated by each particle, and hence the reciprocal of dose to that mass, would be less in ccmpact soft tissue such as skin. annual radiation dose, averaged over $1,000 \mathrm{~g}$ of lung tissue, would be $15 \mathrm{rem}$ ). For convenience, and with no pretence at realism, Table 1 assumes that this MPLB is uniformly distributed in the lungs with varying degrees of aggregation, ranging from a single $37-\mu \mathrm{m}$ particle (actually too big to be respirable) to $\sim 50$ million $0.1-\mu \mathrm{m}$ particles. But each ${ }^{239} \mathrm{PuO}_{2}$ particle, however large, can only irradiate $\sim 50-100$ of the several hundred million alveoli; therefore an MPLB uniformly distributed as particles larger than $\sim 0.15 \mu \mathrm{m}$ must irradiate some lung tissue at more than 15 rem per year and some other tissue not at all. (Nonuniformly distributed particles, as they would be in reality, would give nonuniform doses at even smaller particle sizes.) Nonetheless, the mean lung dose, now used to set the MPLB and MPC would be 15 rem per year in each case shown, and each would be assumed to carry the same cancer risk.

The issue posed by Table 1 is whether intense irradiation of a very small mass of tissue is more carcinogenic than less intense irradiation of a proportionately larger mass; that is, if a given amount of intense irradiation is distributed more evenly over lung tissue, whether the greater number of cells at risk exactly compensates for the smaller risk per cell. An hypothesis that this were untrue would imply that carcinogenesis is not linearly proportional to dose at very high dose levels because intense local irradiation can bring to the fore some new mechanism(s) absent or unimportant at lower doses. (Loosely speaking, this is a "threshold" theory, but one applying to high doses, not low ones: people who argue for non-linearity in the low-level radiation controversy tend to argue against it in the hot-particle controversy.)

Such a high-dose mechanism might involve (for example) carcinogenic or contributory chemical activity by fragments of killed, fibrotic, liquefied, or otherwise seriously disrupted cells, rather than (or in addition to) "primary" or direct carcinogenesis by disruption of, say, a chromosome. Such a "secondary" mechanism may coexist with other mechanisms, is plausible, and has respected adherents, but has not been directly demonstrated. Neither, however, has any other proposed mechanism of radiation carcinogenesis, primary or secondary.

The International Commission on Radiological Protection (ICRP) have sometimes had to introduce a Distribution Factor into the rad-to-rem conversion to allow for inhomogeneous doses within a "critical organ". Thus the dose limit for bone is based on uniformly deposited ${ }^{226} \mathrm{Ra}$; but $\mathrm{Pu}$, collecting at the bone surface, is considered $\sim 5$ times as radiotoxic, so a Distribution Factor of 5 is used to compute $\mathrm{Pu}$ bone dose. Likewise, IAEA TR-142 (1973) reports 
that "because it had been recognised that the dose distribution [from inhaled radon daughters] in the respiratory tract was inhomogeneous and the maximum alpha dose was delivered to the bronchial epithelium" rather than to the whole lung, the ICRP suggested a Distribution Factor of 10, owing to evidence that uranium miners were getting lung cancer more often than the average-lung-dose model had predicted. So far, no Distribution Factor $>1$ has been applied to $\mathrm{Pu}$ in lung; and NRDC suggest, in effect, that the "critical organ" limited to 15 rem per year should be not the complete pair of lungs, but rather the tissue irradiated within it. Otherwise, using the doseaveraging concept, a $\mathrm{Pu}$ particle in beagle lung would be 8 times as carcinogenic as the same particle in human lung (neglecting species differences) simply because human lung is 8 times as large. Indeed, if each of two men had an identical $\mathrm{Pu}$ particle in his left lung but the right lung of one man had previously been removed, his computed risk of lung cancer from the particle would be twice that of the other man! This reductio ad absurdum shows the artificiality of averaging very localised doses over entire organs.

The ICRP have explicitly declined to offer guidance in this area: as the NRPB state, "radiological protection standards cannot be applied to small volumes as they have been derived from observation of biological effects in the whole body or in body organs." Experiments have shown, however, that intense irradiation of, or other insults to, small amounts of various tissues (including bronchial and lung tissue) can produce tumours or other lesions showing cellular changes characteristic of precancerous lesions. Indeed, $\mathrm{Pu}$ particles in rat lung have been shown to cause lesions similar to one observed around a $5-\mathrm{nCi}{ }^{239} \mathrm{Pu}$ particle excised in 1962 from a worker's palm: this lesion showed "severe" changes similar "to known precancerous epidermal cytological changes". There is strong evidence, too, associating a fatal soft-tissue sarcoma in a US worker with entry, through some minute cut, of $\mathrm{Pu}$ solution that had spilled on his hand. Thus cancer, or lesions suggestive of eventual cancer, is demonstrably induced in both man and laboratory animals by very intense irradiation of very small amounts of various tissues, including rat lung. But does the incidence of such effects exceed that observed for lower intensities applied to proportionately larger amounts of tissue?

No direct answer is available for inhaled insoluble Pu. Disquieting long-term experiments with beagles have shown that -almost $100 \%$ of dogs inhaling $\mathrm{Pu}$ doses down to $200 \mathrm{nCi}\left(\sim 10^{7}\right.$ particles) get lung cancer within $\sim 11$ years, but experiments with lower doses will not yield data for many years. The evidence

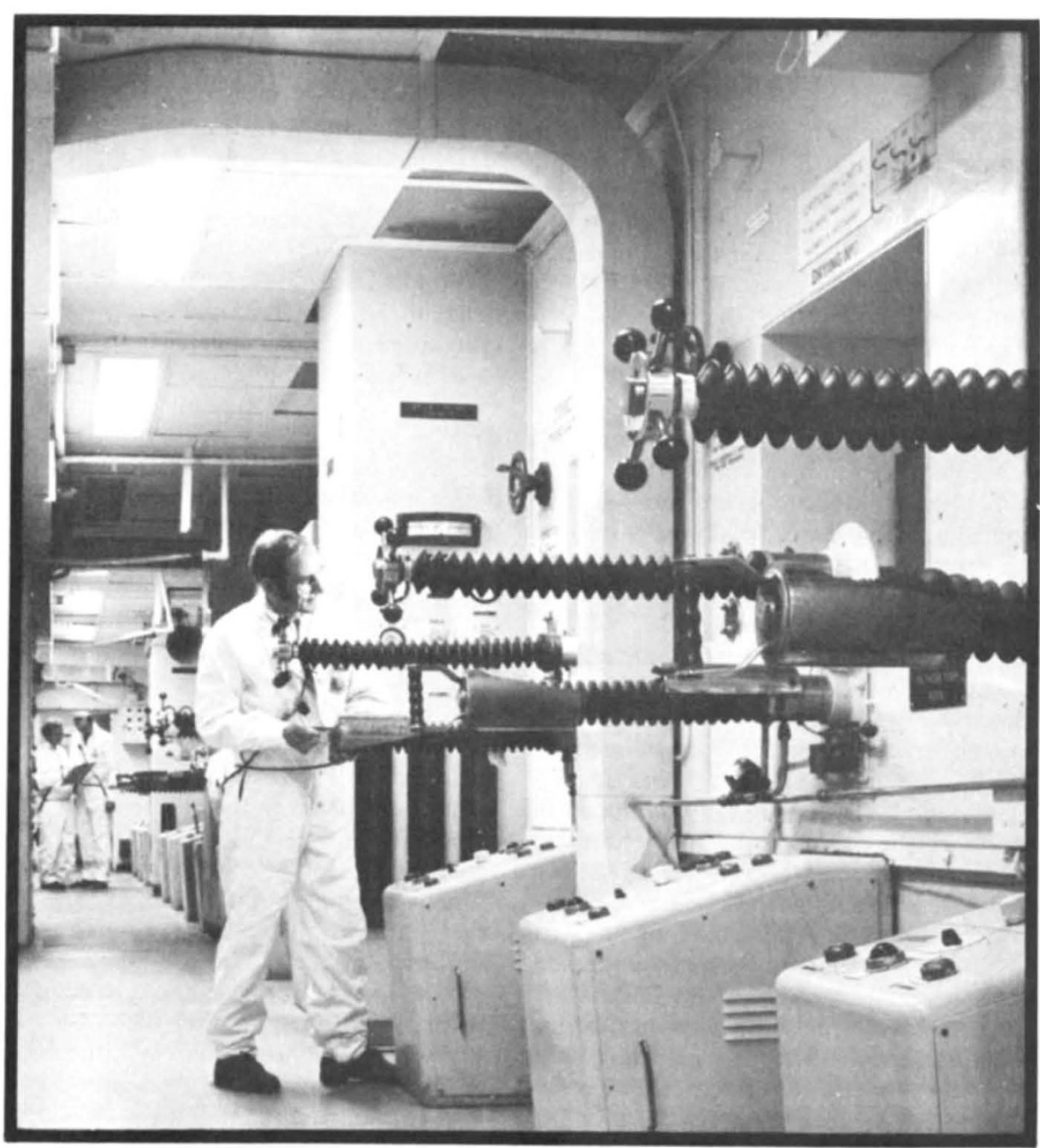

Windscale: workers on the plutonium finishing line

must therefore be indirect, so one must decide what evidence is relevant and hence exactly what is a "hot particle". The February 1974 NRDC definition, based largely on work by Geesaman, is an alpha-emitting "particle in the deep respiratory tissue of such activity as to expose the surrounding lung tissue to a dose of at least 1,000 rem in 1 year." New data (including human autopsy data) discussed by NRDC in a February 1975 paper suggest that a lower limit for hot-particle activity might be anywhere from $0.07 \mathrm{pCi}$ (derived from the $1 \mathrm{krem}$ per year criterion) to perhaps $4.2 \mathrm{pCi}$, -a four-fold range of particle diameterdepending on the data and degree of conservatism chosen. NRDC also prefer to define the limit not by calculated local dose but by particle activity, which can be correlated with observable phenomena: some levels of activity produce fibrotic lesions whilst others do not.

Clearly it is necessary to assume some lower activity limit distinguishing the hotparticle carcinogenic mechanism from that of uniform dose, and any such postulated limit must have a certain arbitrariness which invites challenge. (The limit may not in reality be so clearly defined.) Suggestively, the 0.07-pCi limiting activity originally proposed by NRDC is substantially above the level at which uniformly distributed particles totalling 1 MPLB cannot irradiate the whole lung (Table 1).

No quibbling can arise over whether a particle is a hot particle if its activity is orders of magnitude too low to cause observable discrete lesions. The particles used in many experiments, those produced by fallout from nuclear testing, and those inhaled by the often-cited 25 Manhattan Project workers are all in this category and hence cannot be used to test the NRDC hypothesis. At least 25 other workers did inhale hot particles from the 1965 Rocky Flats fire, and linear extrapolation from the beagle data suggests that these men are likely to develop lung cancer after an unknown induction period, probably of several decades.

Many critics of the hot-particle hypothesis do not state whether the experiments they cite involve hot particles. For example, the NRPB's dismissive review of the NRDC hypothesis relies heavily on an unpublished paper of Lafuma, comparing the incidence of lung cancer in rats after inhalation of "particulate" ${ }^{239} \mathrm{Pu}$ or "diffuse" ${ }^{242} \mathrm{Cm}$. NRPB claim a lower incidence for the former doses, but give no data, and one is entitled to wonder whether the ${ }^{239} \mathrm{Pu}$ was in the form of hot particles, what the doses were, whether the lifespan of the rats exceeded the 
induction time of the effects sought, what allowance was made for the 23-week halflife of ${ }^{242} \mathrm{Cm}$ and so on. Inquiries to NRPB in January, 1975 elicited the astonishing reply that such basic data as particle size and activity were not in Lafuma's paper and must be sought from him. (An immediate letter to that end has had no reply at this writing.) So it is not clear whether the NRPB's crucial experiments involve hot particles at all.

Even if they do, the data are still consistent with the hot-particle hypothesis. Experiments suggesting that tumour incidence per $\mathrm{nCi}$ is greater with uniform than with discrete dose distribution-or the opposite, as both can be shown-are not relevant to, nor a test of, the hot-particle hypothesis: the latter bears on the risk per hot particle, not the risk per $\mathrm{nCi}$. If the hypothesis is right, then increasing the activity of a hot particle-one that is already large enough to bring into play the postulated high-dose mechanism of carcinogenesis--only further disrupts already-killed cells and does not significantly increase the risk from that particle, though it reduces the apparent risk per $\mathrm{nCi}$. Thus the hypothesis already contains the concept of "overkill" or "wasted radiation". Table 2 shows six hypothetical experiments devised by NRDC: $A-D$ suggest that discrete dose is less hazardous than uniform dose, $E-F$ suggest the opposite, and $A-F$ are all consistent with the hot-particle hypothesis.

The NRDC rebuttal of WASH-1320 appears to show that all experiments there cited are either irrelevant to the hot-particle hypothesis or consistent with it. The irrelevance arises because for example, experiments do not involve insoluble hot particles in lung, or use short-half-life actinides, or show rapid lung clearance, or have been interpreted on a risk per $\mathrm{nCi}$ basis, or use animals that do not survive the induction period, or do not wait for it to elapse, or mask the lung-cancer response with other pathological conditions, or do not involve significant samples.

The MRC report, apparently initiated by the NRDC petition, cites it as the work of "an organisation called the Environmental Defense Research Council concerned with problems of pollution in general" (wrong on both counts) and, like the NRPB report, ignores the two 1974 NRDC rebuttals which follow up the original petition and anticipate the MRC and NRPB arguments. (The MRC report does not even cite the two AEC responses to the NRDC petition.) By failing to read these papers or to contact NRDC, the MRC and NRPB have lost an opportunity to raise arguments not already canvassed. They have stressed the quantitative uncertainties (limiting particle activity and risk per particle) but have not disproved the basic thesis. Instead, they prefer to argue that "there is no evidence" for the NRDC hypothesis- whilst ignoring much of the evidence NRDC cite in their original paper, notably reports of $\mathrm{Pu}$-induced lesions in both rats and man. But the "no evidence" argument cuts both ways. There has been no concerted effort in Britain to imitate the new US Transuranium Registry of radiation workers, which it is hoped will eventually find the epidemiological evidence if it exists. To argue from the general absence of evidence (for which one has not looked) that there is no evidence for one's opponent's point of view is to write with an economy of truth.

The main drawback of the hot-particle hypothesis seems to be that it requires one to assume an unproven and somewhat vague cellular mechanism of radiation carcinogenesis operating at high dose levels. Yet critics of the hypothesis have been unable to demonstrate any mechanism underlying their own theories, and until they have done so, it is hard to see how other mechanisms can be definitely excluded. The resulting scientific uncertainty, and its likely persistence, raise an important ethical and political issue. We have an hypothesis, neither proved nor disproved, implying that the MPLB and $\mathrm{MPC}_{\mathrm{a}}$ for insoluble aerosols of $\mathrm{Pu}$ and similar actinides--inherent in a largescale fission economy-should be reduced by orders of magnitude. We have two choices: (1) ignore the hypothesis as unproven, continue our research, continue to expose some radiation workers to hot particles, assume that these are innocuous, and proceed to make the world's energy supplies dependent on annual flows of hundreds of tons of $\mathrm{Pu}$; or (2) provisionally adopt the hypothesis until it is disproven, tighten standards accordingly, and thus act with the caution, prudence, and conservatism whichwe are told-are the basic principles of radiation protection. So far, regulatory bodies have taken the first course, gambling that exposed $\mathrm{Pu}$ workers will not begin, a decade or two hence, to show excess lung cancers as some analysts claim they are now beginning to show excess leukaemias.

The reason for this gamble may be related to a little-known fact: that even the present MPLB and MPC ${ }_{a}$ for insoluble $\mathrm{Pu}$ are near the limits of practical detectability, not because we lack sensitive instruments but for fundamental statistical reasons. It is not clear that present monitoring methods at, say, Windscale can demonstrate compliance even with present standards: a "puff" release from, say, a defective glove-box may be hidden in the 24-hour integration period of air samplers, and even a real-time radiation monitor could probably not detect significant $\mathrm{Pu}$ levels in a typical room before most of the air in it had been inhaled or changed. If the standards became much stricter, compliance could never be shown and the standards would have no operational meaning. Whether a Pu-fuelled industry could then operate would depend on whether the burden were on it to prove compliance (impossible) or on others to prove non-compliance (presumptive but debatable) and on how strictly the relevant duties were construed.

The questions of monitoring and enforcement are not academic; they are the crux of the matter, for a few decades from now the growing gap of magnitudes between world Pu stocks (thousands of tons) and the amount which can produce a nuclear explosion (a few $\mathrm{kg}$ ) or give a beagle lung cancer (a few $\mu \mathrm{g}$ ) will require impressively and increasingly diligent care to prevent releases through accident or malice. Pu metal, nitride and carbide can be pyrophoric, burning to respirable $\mathrm{PuO}_{2}$; this insoluble ceramic is itself present in advanced nuclear fuel cycles, can pass in respirable sizes through high-efficiency filters, and has contaminated dozens of $\mathrm{Pu}$ workers in accidents here and abroad. $\mathrm{Pu}$ fires have released tens or hundreds of grams of $\mathrm{PuO}_{2}$ from US facilities and far more within them. $\mathrm{Pu}$ could be released by fast-reactor accidents, weapons explosions, deliberate dispersal, or chemical or microbial action on Pu-bearing wastes (like the dilute alpha wastes buried at Drigg near Windscale). Biological pathways for reconcentration of $\mathrm{Pu}$-now discharged in hundreds of $\mathrm{Ci}$ per year from Windscale into the Irish Sea-may exceed those now known, as Pu chemistry is very complex. Since the half-life of ${ }^{239} \mathrm{Pu}$ is 24,390 years, and since fallen-out aerosols may become resuspended (data on this vary over 11 orders of magnitude), dispersal may be essentially permanent. In an energy economy based on hundreds, then thousands, of fast breeder reactors, each containing several tons of $\mathrm{Pu}$, even if $\mathrm{Pu}$ escape were controlled with the most exquisite care, the unavoidable limits of in vivo and environmental measurement would make it impossible to tell whether we were meeting stricter $\mathrm{Pu}$ standards or not. We could only tell from increased lung cancers-decades after the irrecoverable releases had occurred.

Table 2 Some hypothetical hot-particle experiments

\begin{tabular}{|c|c|c|c|c|c|c|}
\hline Experiment & $A$ & $B$ & $C$ & $D$ & $E$ & $F$ \\
\hline Number of hot particles & 6000 & 4000 & 2000 & 200 & 6000 & 4000 \\
\hline Total activity (nCi) & 12 & 12 & 12 & 12 & 12 & 6 \\
\hline $\mathrm{pCi} /$ particle & 2 & 3 & 6 & 60 & 2 & 1.5 \\
\hline Number of tumours observed & 3 & 2 & 1 & 0 & 3 & 2 \\
\hline Tumours $/ \mathrm{nCi}$ & 0.25 & 0.17 & 0.08 & 0.00 & 0.25 & 0.33 \\
\hline Tumours/particle & 0.0005 & 0.0005 & 0.0005 & - & 0.0005 & 0.0005 \\
\hline
\end{tabular}

